\title{
Characterizations of UV-Curable Montmorillonite/Epoxy Nanocomposites Prepared by a Hybrid of Chemical Dispersion and Planetary Mechanical Milling Process
}

\author{
Heh-Chang Huang, ${ }^{1}$ Shu-Ping Huang, ${ }^{1}$ Tsung-Eong Hsieh, ${ }^{1}$ Chin-Hsin Chen ${ }^{2}$ \\ ${ }^{1}$ Department of Materials Science and Engineering, National Chiao Tung University, \\ Hsinchu, Taiwan 30010, Republic of China \\ ${ }^{2}$ Microelectronics and Information Systems Research Center, National Chiao Tung University, \\ Hsinchu, Taiwan 30010, Republic of China
}

Received 30 November 2010; accepted 20 May 2011

DOI 10.1002/app.34988

Published online 6 September 2011 in Wiley Online Library (wileyonlinelibrary.com).

\begin{abstract}
Ammonium salt, [2-(methacyloyloxy)ethyl] trimethylammonium methyl sulfate (MAOTMA), was adopted to modify montmorillonite (MMT) via the cationic exchange process in an acidic environment and a successful enlargement of MMT lamellar spacing from 1.18 to $1.39 \mathrm{~nm}$ was identified by x-ray diffraction and Fouriertransform infrared spectroscopy. Accordingly, UV-curable epoxy nanocomposite resins containing various amounts of MAOTMA-modified MMT were prepared with the aid of a planetary mechanical milling process. Transmission electron microscopy revealed a uniform dispersion of exfoliated MMT lamella in epoxy matrix, leading to the distinct improvements on thermal stability and thermal
\end{abstract}

expansion property of nanocomposite samples. Calcium (Ca) test indicated that the good dispersion of MMT fillers in polymer effectively suppresses the moisture permeation in sample matrix. More than twofold increment on retention time of $5 \%$ Ca hydrolysis and $20 \%$ improvement on the permeation kinetic parameters were observed in the nanocomposite sample containing $8 \mathrm{wt} \%$ MMT in comparison with the pristine epoxy sample. (C) 2011 Wiley Periodicals, Inc. J Appl Polym Sci 123: 3199-3207, 2012

Key words: clay; dispersions; gas permeation; microstructure; nanocomposites

\section{INTRODUCTION}

Organic/inorganic nanocomposites have been widely investigated in past decade due to their versatile optical, mechanical, thermal, electrical, and transport properties. ${ }^{1-7}$ Owing to the advantages of high aspect ratios, high tensile modulus, low cost, and low thermal expansion property, lamellar silicate clays are the popular inorganic components of nanocomposites. However, it is difficult to disperse natural clays in polymers due to the high van der Waals force between clay lamella and the incompatibility of hydrophilic clays with hydrophobic polymers. Modification of clays is thus a crucial process in the preparation of clay/polymer composites with fine dispersion structure. ${ }^{7-13}$ Usuki et al. adopted $\omega$ amino acid to replace sodium ions in clay via a cationic exchange process and then used $\varepsilon$-caprolactam to promote the polymerization in between the orga-

\footnotetext{
Correspondence to: T.-E. Hsieh (tehsieh@mail.nctu.edu. tw).

Contract grant sponsor: National Science Council (NSC), Taiwan, R.O.C.; contract grant number: NSC96-2221-E-009010 .

Journal of Applied Polymer Science, Vol. 123, 3199-3207 (2012) (C) 2011 Wiley Periodicals, Inc.
}

noclay lamella to obtain the clay/nylon-6 nanocomposites. ${ }^{8,14} \mathrm{Zhu}$ et al. synthesized the phosphonium and ammonium salts to modify the montmorillonite (MMT) and then polymerized in MMT gallery to achieve the MMT/styrene nanocomposites. ${ }^{10} \mathrm{~A}$ combination of ammonium salt and silane coupling agent to graft the clay interfaces and bond with polymer via polymerization to form the nanocomposites were also reported. ${ }^{11,12}$ Modification of clays by utilizing ammonium or phosphonium salts not only enlarges the lamellar spacing but also improves the polarity of clays, leading to the easy entrance of monomers into clay gallery so as to achieve the nanocomposites with uniformly dispersed microstructure. Review on the modification of clays, dispersion mechanisms and relevant applications can be found, for instance, in de Pavia et al.'s article. ${ }^{13}$

The dispersion morphology is known to correlate with the physical/chemical properties of clay/polymer nanocomposites. Yano et al. reported the exfoliated clay in polyimide could effectively suppress the gas permeation and the coefficients of thermal expansion (CTEs) of samples. ${ }^{15,16}$ Exfoliated MMT/ polystyrene and $\mathrm{MMT} /$ polypropylene nanocomposites might reduce the flammability. ${ }^{10,17}$ Chen et al. demonstrated the improvements of mechanical and thermal properties in exfoliated MMT/polyurethane 
nanocomposites. ${ }^{18}$ These researches clearly illustrated a uniform dispersion of clay lamella in polymeric matrix is the key to the clay/polymer nanocomposites with low filler content and satisfactory performance.

Epoxy is the most common polymeric resin for electronic and optoelectronic industries due to its good thermal stability, high transparency, good adhesive strength, and moderate viscosity. For electronic packaging, satisfactory sealing capability is an essential concern for sealing adhesives. Further, the optoelectronic products such as organic light emitting devices (OLEDs) require the sealing process at temperatures below $100^{\circ} \mathrm{C}$ to avoid the degradation of light-emitting materials. Photo-curable nanocomposite sealing resins with low permeation property hence become the primary choice for such an application. As a matter of fact, UV-curable polymerization is an environmentally friendly process with fast, solvent-free, and low energy consumption features. ${ }^{19,20}$ Previous studies ${ }^{20-23}$ on the epoxy/clay nanocomposites have demonstrated the enhancements on thermal, mechanical, and permeation properties without the slow-down of photo-polymerization kinetics. ${ }^{20,21}$ In this work, the modification of MMT utilizing the ammonium salt, [2-(methacyloyloxy)ethyl] trimethylammonium methyl sulfate (MAOTMA), is presented and the UV-curable epoxy nanocomposite resins containing MAOTMA-modified MMT/epoxy are prepared with the aid of planetary mechanical milling process. Accordingly, the correlation of microstructure to thermal and gas permeation properties of nanocomposites is presented as follows.

\section{EXPERIMENTAL}

\section{Materials}

The high-purity $\mathrm{Na}^{+}$-MMT clay powder (PK802) with the cationic exchange capacity (CEC) of 95 $\mathrm{mEq} / 100 \mathrm{~g}$ and initial size in the range of $0.03-0.07$ $\mathrm{mm}$ was purchased from Pai Kong Ceramic Material, Taiwan, R.O.C. The photo-initiator, iodonium (4-methylphenyl) [4-(2-methylpropyl) phenyl]-hexafluoro-phosphate (1-) (IRGACURE 250), and the photo-sensitizer, mixture of 2-isopropylthioxanthone and 4-isopropylthioxanthone (DAROCUR ITX), were purchased from Ciba. Cationic photo-polymerization process was adopted for the preparation of UV-curable nanocomposite resin sample since it provides high adhesive strength, low shrinkage, and good chemical resistance. Note that the utilization of photo-sensitizer containing above chemicals was to accelerate the rate of photo-polymerization. ${ }^{24}$ Clay modification agent, ammonium salt solution (aqueous solution containing 80 wt \% MAOTMA), and

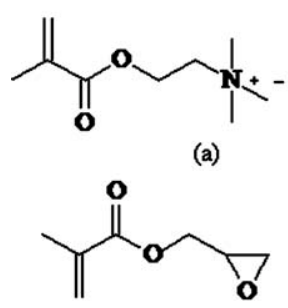

(c)

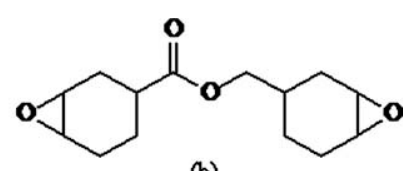

(b)

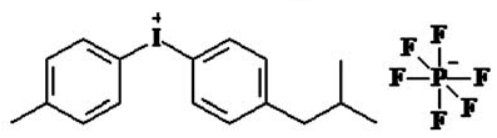

(d)

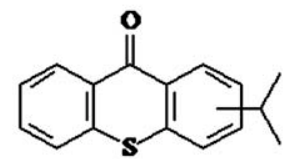

(e)
Figure 1 Molecular structures of (a) MAOTMA modification agent, (b) ECC epoxy monomers (c) SR370 epoxy monomers (d) IRGACURE 250 cationic photo-initiator and (e) DAROCUR ITX photosensitizer.

the epoxy monomer, 3,4-epoxycyclohexylmethyl 3,4epoxycyclohexane carboxylate (ECC), were purchased from Aldrich. Such a low-viscosity epoxy precursor was chosen due to its compatibility with subsequent mechanical milling process. Another epoxy monomer, glycidyl methacrylate (SR379), supplied by Chembridge was adopted since its low-molecular-volume feature allows the easy entrance of organic component into MAOTMA-modified MMT lamella during the mechanical milling process. Hydrochloric acid $(\mathrm{HCl})$ for $\mathrm{pH}$ value adjustment was purchased from SHOWA. All reagents were used directly without further purification and their molecular structures are separately depicted in Figure 1.

\section{Modification of MMT by MAOTMA}

First, $5 \mathrm{~g}$ of MMT was slowly added into $500 \mathrm{~mL}$ of de-ionic water at $80^{\circ} \mathrm{C}$. Subsequently, $0.1 \mathrm{~N}$ of $\mathrm{HCl}$ solution was added under vigorous stirring so as to adjust the $\mathrm{pH}$ value of MMT aqueous solution to the value of 4 and to achieve the swelling of MMT lamella. Afterward, $3.38 \mathrm{~g}$ of MAOTMA was added to above solution at $80^{\circ} \mathrm{C}$ while an appropriate amount of $\mathrm{HCl}$ solution was added so as to maintain the $\mathrm{pH}$ value of the mixture at 4 . The reactions were allowed at the same temperature for $4 \mathrm{~h}$ under vigorous stirring and then the mixture was cooled down to room temperature. We note the reacting time of modification process was determined by the ICP-MS (inductively coupled plasma mass spectroscopy; Perkin Elmer, SCIEX ELAN 5000) measurement which monitors the residual amount change of sodium cation, $\mathrm{Na}\left(\mathrm{H}_{2} \mathrm{O}\right)_{4}{ }^{+}$, in the clay samples. At the present processing condition, it required more than $3 \mathrm{~h}$ to reduce the residual amount of $\mathrm{Na}\left(\mathrm{H}_{2} \mathrm{O}\right)_{4}{ }^{+}$from $4.42 \%$ for the pristine MMT to $0.022 \%$ for the MAOTMA-modified MMT. Hence, 
the MMT modification was allowed at the time span for $4 \mathrm{~h}$. This resulted in the MAOTMA-modified MMT precipitates and supernatant was removed by centrifugation. De-ionic water was added into the MAOTMA-modified MMT precipitates and centrifugation flushing was performed for at least four times until the clean MAOTMA-modified MMT precipitates were obtained. The MAOTMA-modified MMT was then dried and screened through 200-mesh sieves.

\section{Preparation of MAOTMA-modified MMT/epoxy nanocomposite resins}

Various amounts of MAOTMA-modified MMT, 8.5 $\mathrm{g}$ of ECC and $1.5 \mathrm{~g}$ of SR379 were added in a jug containing zirconia milling balls with $0.5 \mathrm{~mm}$ in diameter. The mechanical milling was achieved by using a planetary mill (RETSCH PM-1000) at the milling rate of $500 \mathrm{rpm}$. There was a 2-min stop interval per 10-min mill and the total milling time span was $36 \mathrm{~h}$. After the milling of MAOTMA-modified MMT and epoxy monomers, $0.3 \mathrm{~g}$ of IRGACURE 250 and $0.1 \mathrm{~g}$ of DAROCUR ITX were added to complete the preparation of UV-curable MAOTMA-modified MMT/epoxy nanocomposite precursors. In this work, the nanocomposite precursors containing 1,5 , and 8 wt $\%$ of MAOTMA-modified MMT were prepared. For the purpose of comparison, the MAOTMA-modified MMT/epoxy nanocomposites were also prepared without the aid of mechanical milling. Photo-polymerization of nanocomposite thin-film samples for subsequent analyses were carried out in an UV oven (ECSUN UC-1000, Taiwan, R.O.C.) at the wavelengths range of 325-385 nm with the curing power $=80 \mathrm{~W}$, curing time $=60-80 \mathrm{~s}$ and distance between light source and reaction apparatus $=15 \mathrm{~cm}$.

\section{Microstructure and physical property characterizations}

The swelling behaviors of MMTs subjected to MAOTMA modification were evaluated by an $x$-ray diffractrometer (XRD, MacScience M18XHF) within $\mathrm{Cu}-K_{\alpha}$ radiation $(\lambda=0.154 \mathrm{~nm})$ at a scan rate of $3^{\circ} /$ min. A Perkin Elmer Spectrum 100 Fourier-transform infrared (FTIR) spectrometer was adopted to characterize the FTIR spectra of MAOTMA-modified MMTs. The amount of sodium cations, $\mathrm{Na}\left(\mathrm{H}_{2} \mathrm{O}\right)_{4}{ }^{+}$, absorbed on MMT lamella removed by the MAOTMA modification was identified by an inductively coupled plasma-mass spectrometer (ICP-MS, Perkin Elmer, SCIEX ELAN 5000). Thermogravimetric analysis (TGA) in a TA Instruments Q500 TGA operating in nitrogen atmosphere at a heating rate of $10^{\circ} \mathrm{C} / \mathrm{min}$ and thermomechanical analysis (TMA) in

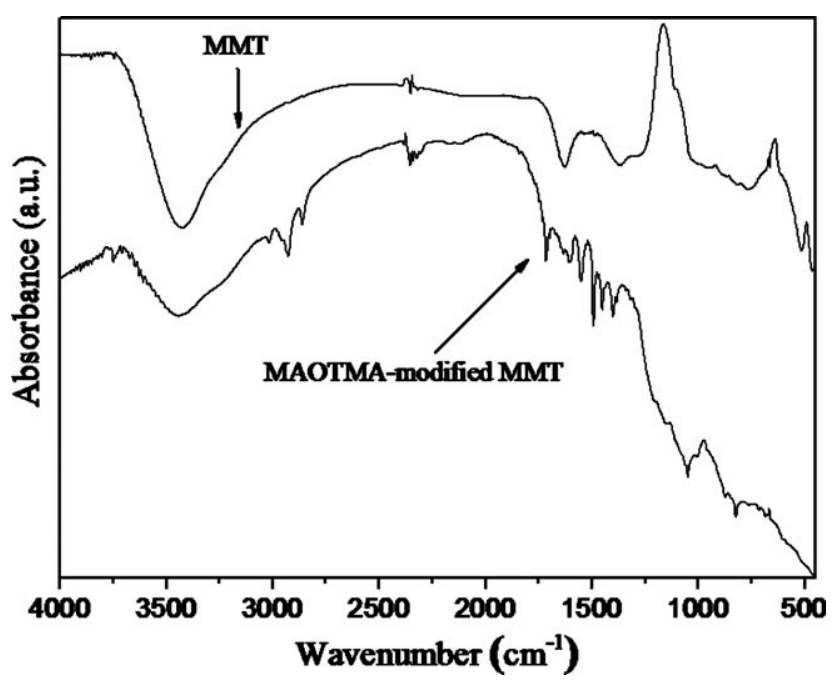

Figure 2 FT-IR spectra of pristine MMT and MAOTMAmodified MMT. Characteristic peak assignments are summarized in Table I.

a DuPont $2940 \mathrm{TMA}$ at a heating rate of $5^{\circ} \mathrm{C} / \mathrm{min}$ were separately performed to deduce the thermal stability and CTE's of nanocomposite samples. Microstructures of nanocomposites were examined by transmission electron microscopy (TEM, Philips Tecnai 20) operating at accelerating voltage of 200 $\mathrm{kV}$ and the TEM samples were prepared by using an ultramicrotomy (Leica UltraCut E).

\section{Permeation test}

Calcium (Ca) test was adopted to evaluate the permeation property of nanocomposite samples. ${ }^{25}$ First, a 200-nm thick Ca thin film was deposited on glass substrate by thermal evaporation. By dispersing the nanocomposite resin precursor around the periphery of glass substrate, another glass lid was immediately placed on. After completing the UV-curing of nanocomposite resin, the sealed sample was transferred to a bench-top test chamber (Terchy, HRM-120) with the humidity fixed at $90 \% \mathrm{RH}$ and adjustable ambient temperature. The hydrolysis of $\mathrm{Ca}$ layer was evaluated at various time spans according to the method described in Ref. 25 so as to deduce the permeation property of nanocomposite samples.

\section{RESULTS AND DISCUSSION}

\section{Characterizations of MAOTMA-modified MMTs}

Figure 2 presents the FTIR spectra of pristine MMT and MAOTMA-modified MMT and the characteristic peaks assignments for these samples are summarized in Table I. The absorbance peaks of pristine MMT appearing at the wavenumbers of 466,520 , 1037, and $3420 \mathrm{~cm}^{-1}$ correspond to the $\mathrm{Mg}-\mathrm{O}$ stretching, $\mathrm{Al}-\mathrm{O}$ stretching, $\mathrm{Si}-\mathrm{O}$ stretching, and 
TABLE I

Characteristic FTIR Peak Assignments for Pristine MMT and MAOTMA-Modified MMT Deduced from Figure 2

\begin{tabular}{lcc}
\hline Sample & $\begin{array}{c}\text { Functional } \\
\text { group }\end{array}$ & $\begin{array}{c}\text { Wavenumber } \\
\left(\mathrm{cm}^{-1}\right)\end{array}$ \\
\hline Pristine MMT & $\mathrm{Mg}-\mathrm{O}$ & 466 \\
& $\mathrm{Al}-\mathrm{O}$ & 520 \\
& $\mathrm{Si}-\mathrm{O}$ & 1037 \\
MAOTMA-modified & $\mathrm{O}-\mathrm{H}$ & 3420 \\
MMT & $\mathrm{C}=\mathrm{C}$ & 1636 \\
& $\mathrm{C}=\mathrm{O}$ & 1716 \\
& saturated C-H & 2927 and 2861 \\
& unsaturated C-H & 3017 \\
\hline
\end{tabular}

$\mathrm{O}-\mathrm{H}$ stretching, respectively. ${ }^{26}$ As to the MAOTMA-modified MMT, additional peaks, e.g., the absorbance peaks at 1716, 1636, and $3017 \mathrm{~cm}^{-1}$ corresponding to the $\mathrm{C}=\mathrm{O}, \mathrm{C}=\mathrm{C},{ }^{26}$ and unsaturated $\mathrm{C}-\mathrm{H}$ absorption bands emerge. The peaks at 2927 and $2861 \mathrm{~cm}^{-1}$ corresponding to the saturated $\mathrm{C}-\mathrm{H}$ absorption bands are also observed. This indicates the successful attachment of MAOTMA onto MMT lamella.

XRD spectra of pristine MMT and MAOTMAmodified MMT are depicted in Figure 3. Calculation in terms of the Bragg's law indicated that the $d_{100^{-}}$ spacing of MMT lamella is $1.18 \mathrm{~nm}$ while that of MAOTMA-modified MMT increases to $1.39 \mathrm{~nm}$. This illustrates that the sodium cations $\left(\mathrm{Na}\left(\mathrm{H}_{2} \mathrm{O}\right)_{4}{ }^{+}\right)$ initially absorbed on MMT lamella are effectively replaced by MAOTMA so as to result in the swelling of MMT. Figure 4 presents a schematic illustration of such a MMT interlayer modification process. The length of carbon chain of MAOTMA is about 0.73 $\mathrm{nm}$ and $d_{100}$-spacing of pristine MMT is about 1.00 $\mathrm{nm}$. Since the sum of these two values is larger than the experimentally observed $d_{100}$-spacing of MAOTMA-modified MMT (i.e., $1.39 \mathrm{~nm}$ ), the

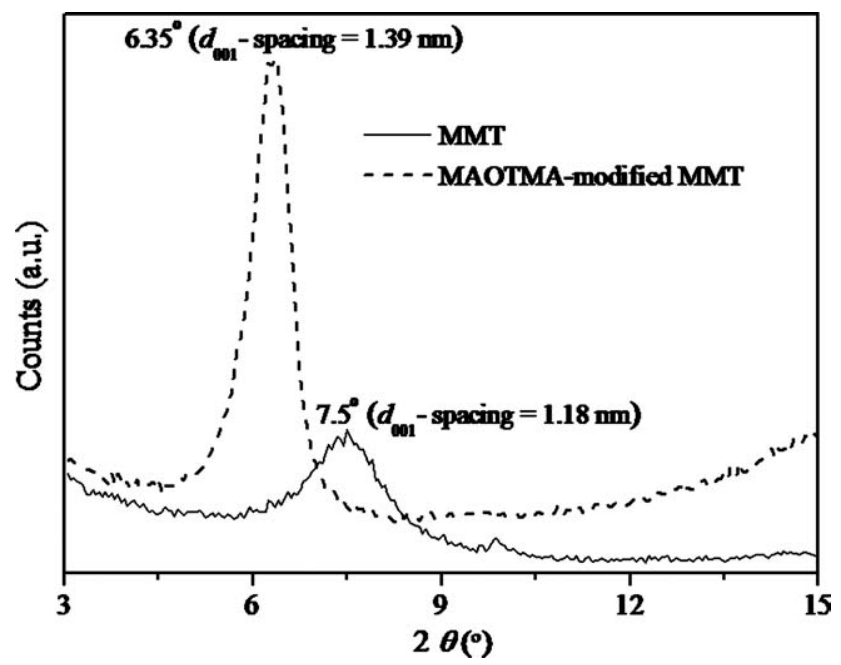

Figure 3 XRD patterns of pristine MMT and MAOTMAmodified MMT. absorbed MAOTMA hence aligns in a tilt manner ${ }^{4,13}$ in between MMT lamella as depicted in Figure 4. We note the successful MMT modification is also evidenced by the result of ICP-MS measurement as described in the section of Experimental.

\section{Characterizations of MAOTMA-modified MMT/epoxy nanocomposites}

Table II summarizes the analytical results of thermal properties of pristine epoxy and nanocomposite samples containing various amounts of MAOTMAmodified MMT deduced by TGA and TMA. It can be readily seen that the 5\%-weight-loss thermal decomposition temperatures $\left(T_{d}\right.$ 's) for nanocomposite samples are always higher than that of pristine epoxy. Further, the value of $T_{d}$ increases with the increase of inorganic filler loading and, in comparison with pristine epoxy, a nearly $30^{\circ} \mathrm{C}$ increment of $T_{d}$ is obtained in the nanocomposite containing $8 \mathrm{wt}$ $\%$ of MAOTMA-modified MMT prepared with the aid of mechanical milling process. The chemical
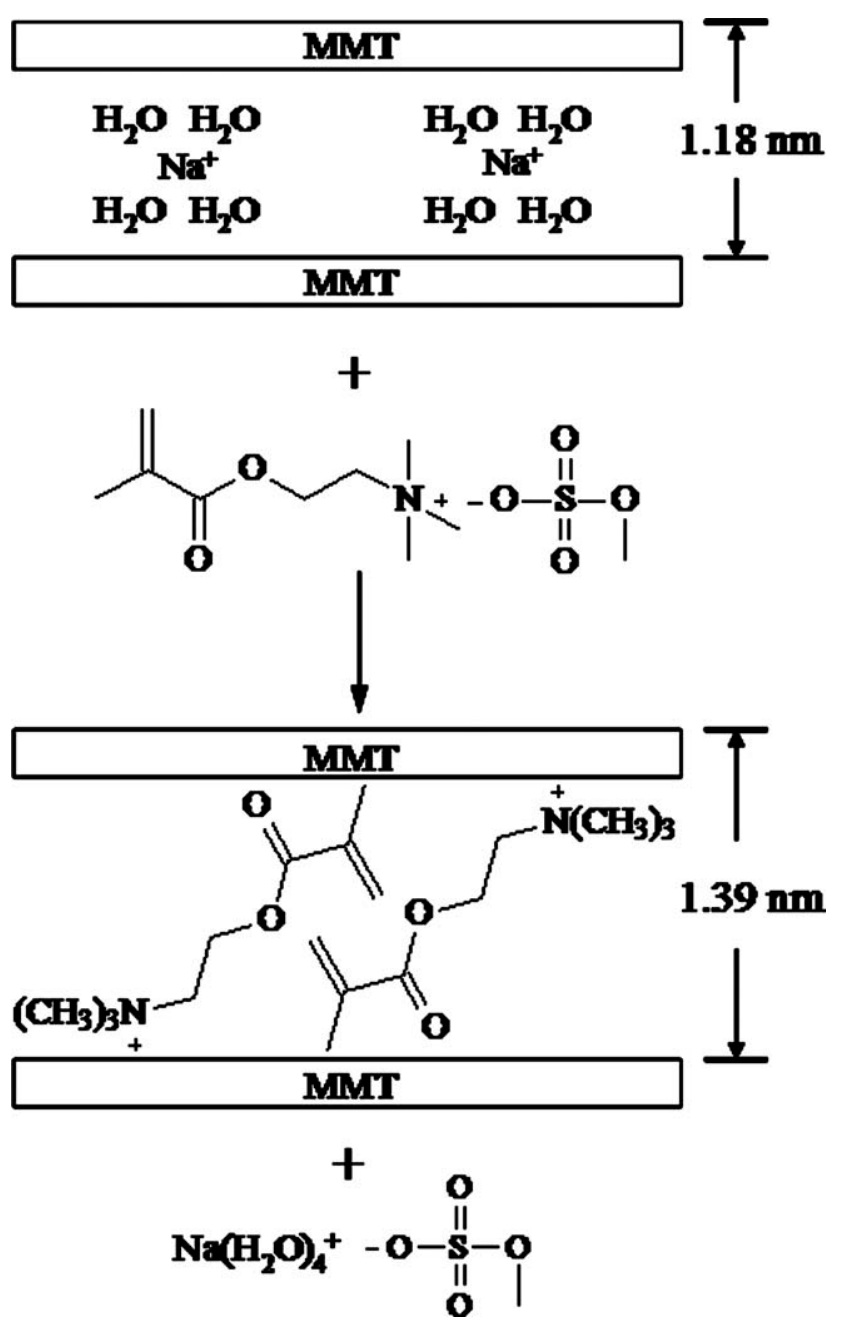

Figure 4 Schematic illustration of intercalation of MAOTMA into MMT lamella. 
TABLE II

A Summary of Thermal Properties for Pristine Epoxy and Nanocomposites Deduced by TGA/TMA Analyses

\begin{tabular}{lcccc}
\hline \multicolumn{1}{c}{ Sample } & $\begin{array}{c}\text { MMT } \\
\text { content } \\
(\text { wt \%) }\end{array}$ & $\begin{array}{c}T_{d} \\
\left({ }^{\circ} \mathrm{C}\right)\end{array}$ & $\begin{array}{c}\text { Residual } \\
\text { weight } \\
(\text { wt. } \%)\end{array}$ & $\begin{array}{c}\mathrm{CTE} \\
\left(\mathrm{ppm} /{ }^{\circ} \mathrm{C}\right)\end{array}$ \\
\hline Pristine epoxy $^{\text {Nanocomposite }}{ }^{\mathrm{a}}$ & 0 & 231.7 & 1.9 & 72 \\
& 1 & 239.8 & 3.4 & 59 \\
Nanocomposite $^{\mathrm{b}}$ & 5 & 252.5 & 7.5 & 43 \\
& 8 & 260.4 & 10.3 & 39 \\
& 5 & 235.7 & 2.3 & 66 \\
& 8 & 245.8 & 6.5 & 56 \\
\end{tabular}

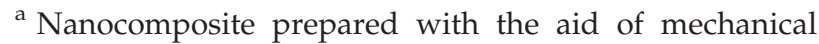
milling.

${ }^{b}$ Nanocomposite prepared without the aid of mechanical milling.

chains formed in between epoxy and MMT lamella are known to restrict segmental motions of polymer. In addition, it has been reported that the MMT lamella may serve as protection layers of polymer chains to block the volatile degraded products in the nanocomposite and thus enhances its thermal stability. ${ }^{27}$ Enhancement of thermal stability of nanocomposites is thus ascribed to the uniform dispersion of exfoliated MMT lamella in epoxy matrix as revealed by TEM characterization reported below.

Table II also illustrates that the incorporation of inorganic fillers effectively suppresses the CTE's of nanocomposites. The CTE of pristine epoxy is 72 $\mathrm{ppm} /{ }^{\circ} \mathrm{C}$ while the CTE of the nanocomposite containing 8 wt \% MAOTMA-modified MMT prepared with the aid of mechanical milling reduces to 39 $\mathrm{ppm} /{ }^{\circ} \mathrm{C}$. In addition to the steric hindrance provided by MMT lamella to block the motions of organic component during heating, MAOTMA-modified MMT possesses $-\mathrm{C}=\mathrm{C}-$ functional group that could bond with epoxy monomer during the photopolymerization. This reinforces the motion blocking capability of inorganic fillers and thus suppresses the CTE's of nanocomposites.

The bottom part of Table II also lists the thermal properties of the nanocomposite samples prepared without the aid of mechanical milling process. A comparison of thermal property data in Table II clearly illustrates that the planetary mechanical milling indeed contributes to the enhancement of thermal properties of nanocomposites. For instance, the mechanical milling raises the $T_{d}$ from 250.3 to $260.4^{\circ} \mathrm{C}$ and suppresses the CTE from 50 to $39 \mathrm{ppm} /$ ${ }^{\circ} \mathrm{C}$ for the sample with $8 \mathrm{wt} \%$ of filler loading. During the milling, the high-speed planetary rotational motion provided a sufficient shear force which effectively enlarged the lamellar spacing of MAOTMAmodified MMT and promotes the entrance of epoxy monomer into the MMT lamella. This benefited not only the formation of organic/inorganic bonds during photo-polymerization, but also the dispersion of MMT lamella in polymer matrix. ${ }^{28-30} \mathrm{~A}$ reinforcement of steric hindrance effects consequently leads to the improvement of thermal properties of nanocomposite samples.

TEM images of nanocomposites containing 1, 5, and 8 wt \% MAOTMA-modified MMT prepared with the aid of planetary mechanical milling are presented in Figure 5. Figure 5(a,c,e) are the low-magnification images whereas Figure $5(b, d, f)$ are the highmagnification images of these samples. For comparison, the samples prepared without the aid of mechanical milling were also analyzed by TEM and the results are given in Figure $6(\mathrm{a}-\mathrm{c})$. As revealed by Figure 5, uniformly dispersed, exfoliated MMT lamellae with an average size about $100 \mathrm{~nm}$ long and $5-10 \mathrm{~nm}$ thick can be readily seen in the samples subjected to mechanical milling. Note that the average filler size was deduced from the measurement of length and thickness of MMT lamella in at least five TEM micrographs. In contrast, relatively thick MMT lamellae with several micrometers in length were observed in the samples without the aid of mechanical milling as illustrated by Figure 6. In addition to the swelling induced by MAOTMA, the planetary mechanical milling adopted in this work considerably contributes to the randomization, size reduction as well as the exfoliation of MMT lamellae in epoxy. Analytical results presented above hence demonstrate the mechanical milling with sufficient shear force can be a valuable tool for the microstructure refinement and physical property enhancement of organic/inorganic nanocomposites.

\section{Permeation properties of MAOTMA-modified MMT/epoxy nanocomposites}

Because of the enhanced physical properties described above, we adopted the nanocomposite resins prepared with the aid of planetary mechanical milling for sample sealing in this part of study. Figure $7(\mathrm{a}-\mathrm{c})$ present the Ca hydrolysis ratios (defined as the area percentage of metallic $\mathrm{Ca}$ layer that has been hydrolyzed into calcium hydroxide, $\left.\mathrm{Ca}(\mathrm{OH})_{2}\right)$ as a function of time for the sealed samples at the test temperatures of 65,75 , and $85^{\circ} \mathrm{C}$, respectively. It is assumed that the hydrolysis of Ca layer obeys the exponential law as follows:

$$
H(t, T)=H(0, T) \exp \left(-\frac{t}{\tau}\right)
$$

where $H(t, T)$ is the Ca hydrolysis ratio at time $t, T$ is the test temperature and $\tau$ is the effective time constant of $\mathrm{Ca}$ hydrolysis with the Arrhenius-type expression $^{31}$ as 

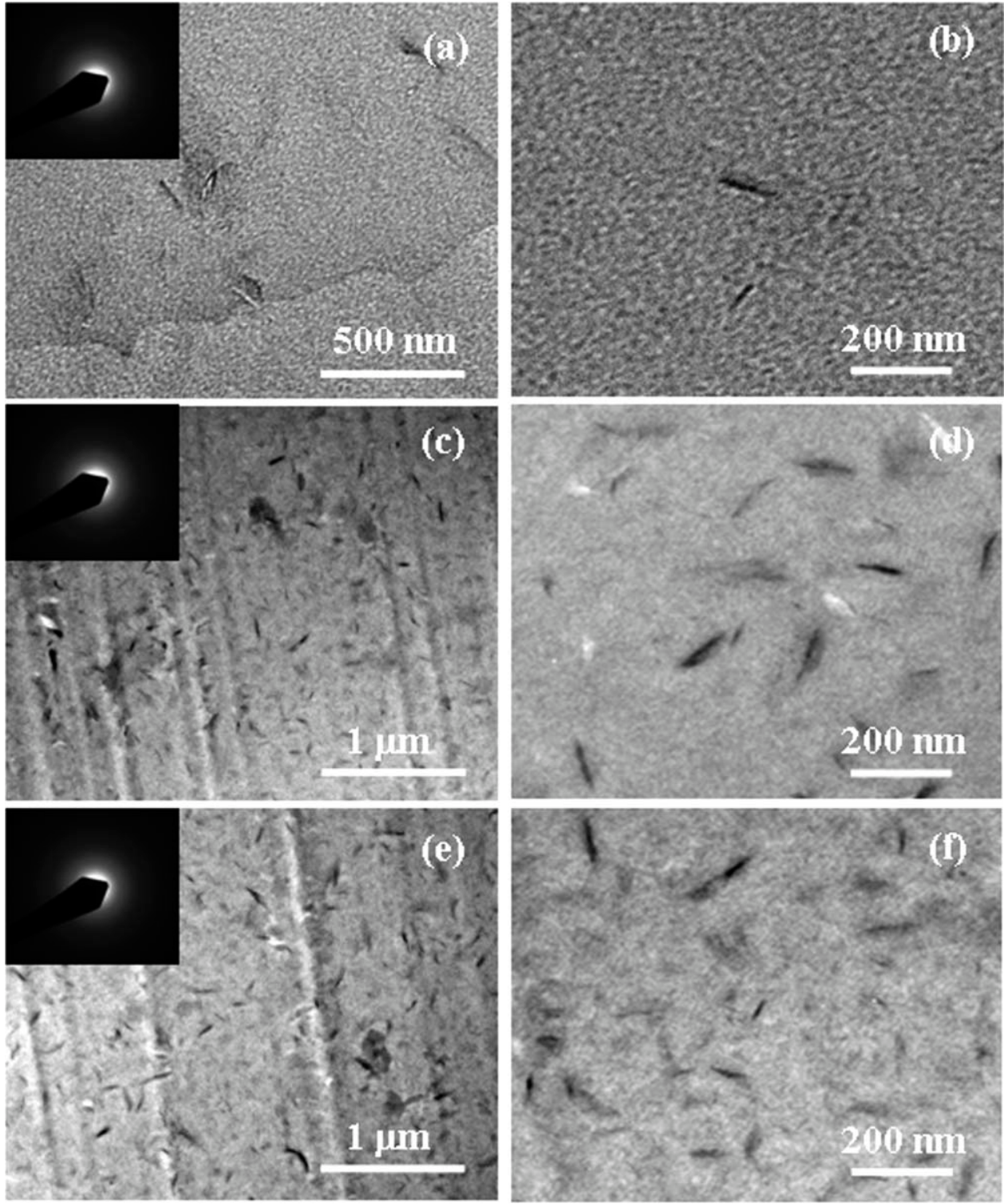

Figure 5 Low-magnification TEM images [(a,c,e)] and high-magnification TEM images [(b,d,f)] of nanocomposites containing 1,5, and 8 wt \% MAOTMA-modified MMT prepared with the aid of mechanical milling. The insets at upper lefthand corners of $(\mathrm{a}, \mathrm{c}, \mathrm{e})$ are the corresponding SAED patterns.

$$
\tau(T)=A \exp \left(\frac{E_{a}}{k_{B} T}\right)
$$

in which $E_{a}$ is the activation energy of Ca hydrolysis and $k_{B}$ is the Boltzmann's constant. Substituting the data presented in Figure $7(\mathrm{a}-\mathrm{c})$ into eq. (1), we obtain the effective time constant $(\tau)$ for each sample at specific test temperature. According to eq. (2), the values of $E_{a}$ can then be extracted from the slopes of $\ln \tau(T)$ versus $1 / T$ plots and our calculation indicated that, as listed in Table III, the values of $E_{a}$ for pristine epoxy and nanocomposites containing 1,5 , and 8 wt \% of MAOTMA-modified MMT are $0.19,0.20$, 0.22 and $0.23 \mathrm{eV}$, respectively. The increase of $E_{a}$ with the increase of inorganic filler content indicates the impedance to moisture diffusion increases in a proportional manner to the inorganic filler loading in sample matrix.

Table III also lists the retention times $t_{R}$ is defined as the time span for metallic Ca film subjected to 5\% hydrolysis) for pristine epoxy and nanocomposites containing various MMT amounts. The values of $t_{R}$ at various temperatures shown in Table III again illustrate that the formation of nanocomposite and increase the inorganic filler loading in polymeric matrix both escalate the barrier to moisture permeation. For instance, the nanocomposite containing $8 \mathrm{wt} \%$ MAOTMA-modified MMT exhibits a more than two-fold improvement on $t_{R}$. Hindrance of moisture permeation in epoxy matrix is ascribed to the tortuous paths established by dispersed MMT lamella in epoxy matrix. ${ }^{15,16}$ 

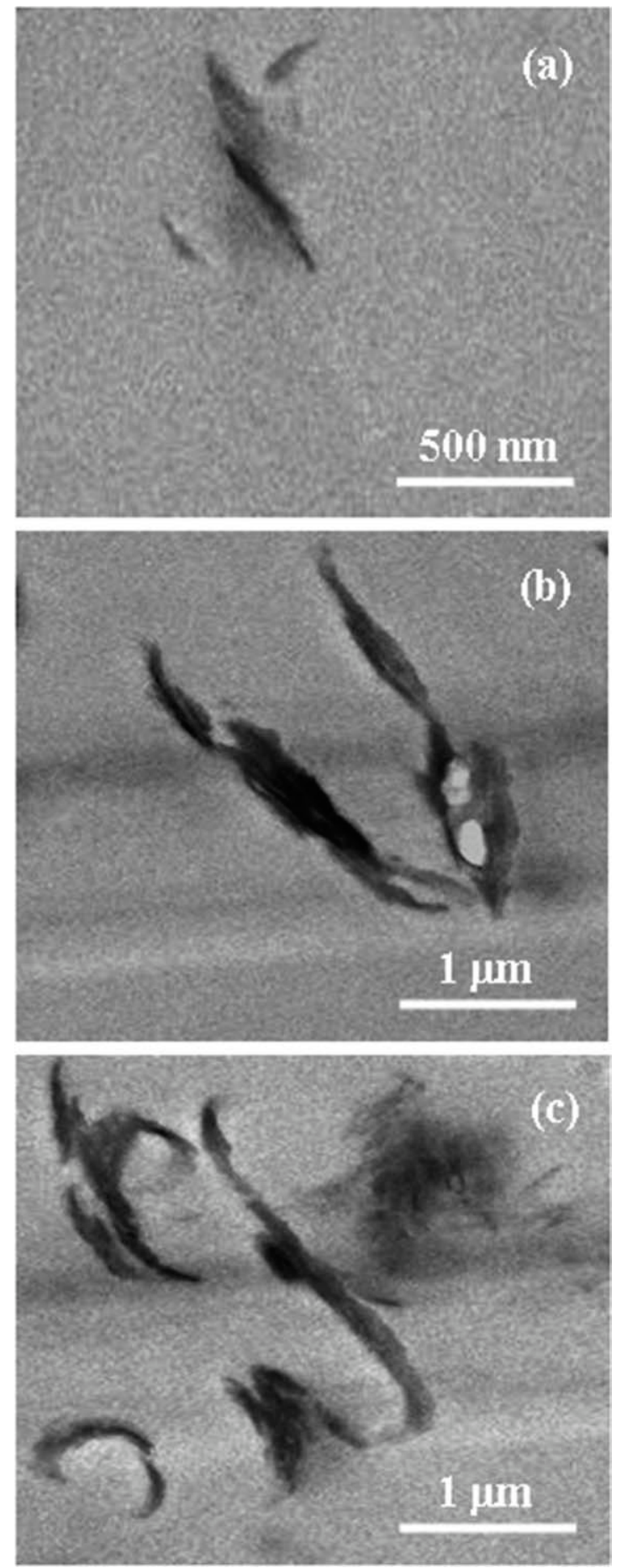

Figure 6 TEM images of nanocomposites containing (a) 1, (b) 5, and (c) $8 \mathrm{wt} \%$ of MAOTMA-modified MMT prepared without the aid of mechanical milling.

Various theoretical models have been proposed to analyze the permeation kinetics in composites containing high-aspect-ratio inorganic fillers. ${ }^{15,16,32-34}$ For randomly dispersed lamellar fillers embedded in polymer matrix, the Bharadwaj's barrier theory derives the expressions for tortuosity factor $\left(\tau_{f}\right)$ and permeability coefficient $\left(P_{c} / P_{p}\right)$ as ${ }^{32}$ :

$$
\begin{gathered}
\tau_{f}=1+\frac{L}{2 W} V_{f} \\
\frac{P_{c}}{P_{P}}=\frac{1-V_{f}}{1+\left(L V_{f} / 2 W\right)(2 / 3)(S+1 / 2)}
\end{gathered}
$$
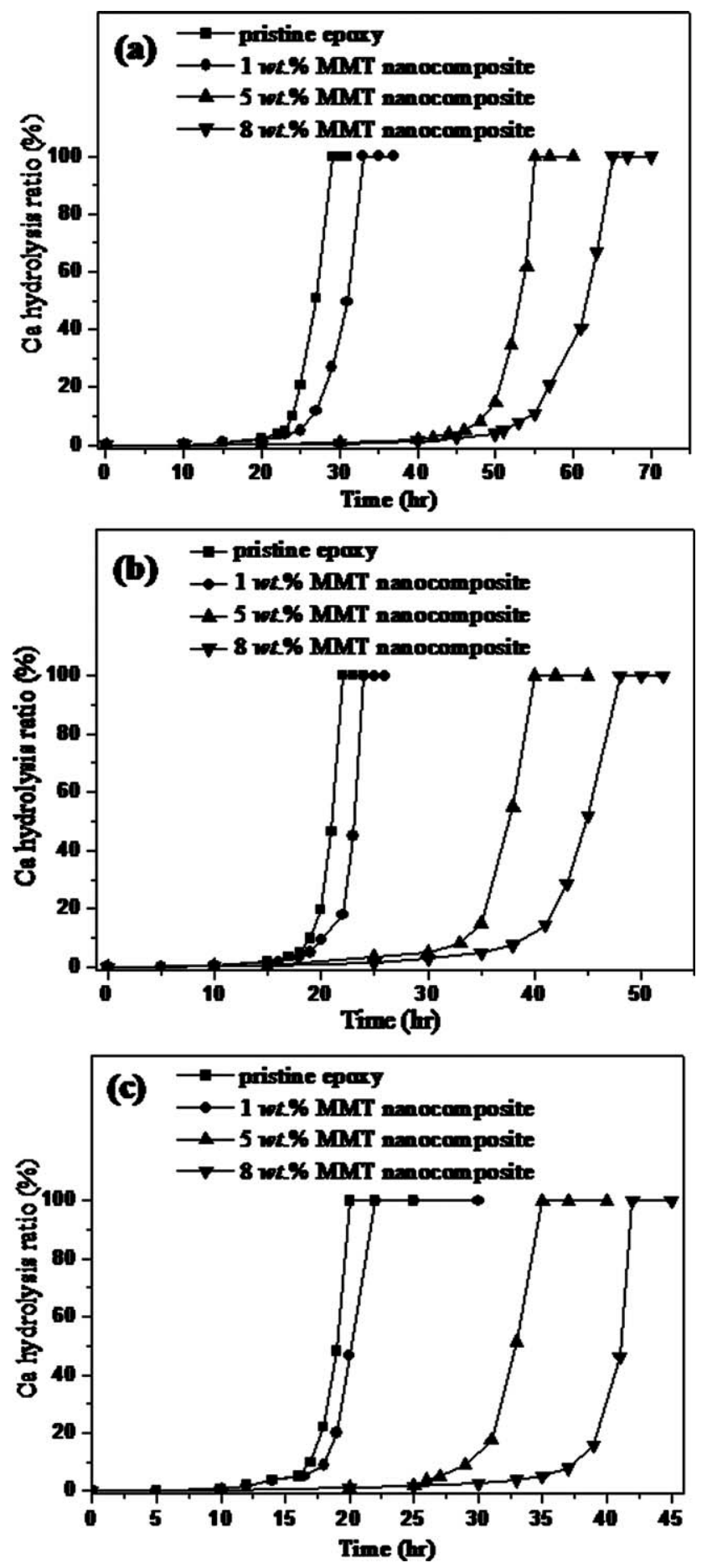

Figure $7 \mathrm{Ca}$ hydrolysis ratios as a function of time for the samples sealed by various nanocomposite resins tested at (a) 65, (b) 75 , and (c) $85^{\circ} \mathrm{C}$. 
where $L$ is the length of lamella, $W$ is the width of lamella, $V_{f}$ is the volume fraction of lamellar filler, $P_{c}$ is the permeability coefficient of composite, $P_{p}$ is the permeability coefficient of pristine polymer. In eq. (4), $S$ is the orientation factor defined as:

$$
S=\frac{1}{2}\left(3 \cos ^{2} \theta-1\right)
$$

where $\theta$ is the angle between preferential orientation direction and lamella normal vector, e.g., $S=1$ (i.e., $\theta$ is equal to $0^{\circ}$ ) means the perfect parallel alignment of platelets while $S=0$ (i.e., $\theta$ is equal to $54.7^{\circ}$ ) means the completely random distribution of platelets in polymeric matrix. As illustrated by TEM images shown in Figure 5, $S=0$ due to the randomly oriented MMT lamella in our nanocomposite samples. Furthermore, the TEM analysis indicates the aspect ratio $(L / W)$ of $1 \mathrm{wt} \%$-MMT sample is about 25 while the aspect ratio for 5 and 8 wt \%MMT samples is about 20. We substituted these data into eqs. (3) and (4) and the calculated values of $t_{f}$ and $P_{c} / P_{p}$ for pristine epoxy and nanocomposites are listed in Table IV. A comparison of permeation kinetic parameters listed in Tables III and IV indicates a good agreement in between the improving trend of permeation property obtained by the Ca test and Bharadwaj's barrier theory. For instance, experimental and theoretical analyses both show an about $20 \%$ improvement on the permeation kinetic parameters for nanocomposite containing $8 \mathrm{wt} \%$ MMT. Finally, analytical results presented above imply, for nanocomposites containing randomly dispersed lamellar fillers, increases of filler loading and its aspect ratio are viable ways to improve the permeation property of samples. However, excessive amount of inorganic filler may escalate the difficulty of dispersion process, degrade the resin adhesion on substrates and might inhibit UV-curing conversion. There is hence a compromise in between resin formula and physical property improvement when preparing the composite sealing resins containing randomly dispersed inorganic fillers.

TABLE III

A List of $E_{a}$ and $t_{R}$ at Various Temperatures for Pristine Epoxy and Nanocomposites Calculated in Terms of the Results of Ca Test

\begin{tabular}{lccccc}
\hline & \multirow{2}{*}{$\begin{array}{c}\text { MMT } \\
\text { content }\end{array}$} & & \multicolumn{3}{c}{$t_{R}(\mathrm{~h})$} \\
\cline { 4 - 6 } Sample & (wt. \%) & $E_{a}(\mathrm{eV})$ & $65^{\circ} \mathrm{C}$ & $75^{\circ} \mathrm{C}$ & $85^{\circ} \mathrm{C}$ \\
\hline Pristine epoxy & 0 & 0.19 & 23.0 & 19.0 & 16.0 \\
Nanocomposite & 1 & 0.20 & 24.5 & 20.0 & 16.5 \\
& 5 & 0.22 & 46.0 & 37.0 & 30.0 \\
& 8 & 0.23 & 51.5 & 41.0 & 33.0 \\
\hline
\end{tabular}

TABLE IV

A List of $\tau_{f}$ and $P_{c} / P_{p}$ for Pristine Epoxy and Nanocomposites Calculated in Terms of Barrier Theory Proposed by Bharadwaj ${ }^{32}$

\begin{tabular}{lccc}
\hline \multicolumn{1}{c}{ Sample } & MMT content (wt. \%) & $\tau_{f}$ & $P_{c} / P_{p}$ \\
\hline Pristine epoxy & 0 & - & 1 \\
Nanocomposite & 1 & 1.03 & 0.96 \\
& 5 & 1.12 & 0.86 \\
& 8 & 1.19 & 0.78 \\
\hline
\end{tabular}

\section{CONCLUSIONS}

This work modifies the MMT lamella by using MAOTMA as dispersant agent in acidic environment and effectively swells the interlayer spacing of MMT from 1.18 to $1.39 \mathrm{~nm}$ as illustrated by FT-IR and XRD analyses. Accordingly, the UV-curable MAOTMA-modified MMT/epoxy nanocomposite samples were prepared with the aid of planetary mechanical milling process. TEM characterization revealed a uniform dispersion of exfoliated MMT lamella in epoxy matrix and the thermal analyses indicated a substantial improvement on thermal properties, e.g., thermal stability and CTE, of nanocomposites. Analytical results illustrated that the planetary mechanical milling process adopted in this work is a valuable tool for microstructure refinement and physical property enhancement of nanocomposite samples. Evaluation of permeation property using Ca test illustrated the formation of nanocomposites effectively hinders the moisture diffusion in polymeric matrix. Good agreement in between experimental and theoretical analyses was also observed in the improving trend of barrier property of nanocomposite samples. An about $20 \%$ improvement on the permeation kinetic parameters of nanocomposite containing 8 wt \% MMT could be achieved in comparison with those of pristine epoxy.

This work is supported by the National Science Council (NSC), Taiwan, R.O.C., under the contract No. NSC96-2221E-009-010. The support of XRD analysis by Dr. Chia-Hung $\mathrm{Hsu}$ at National Synchrotron Radiation Research Center (NSRRC) and Ca test by Mr. Kuan-Heng Lin at Institute of Display, National Chiao Tung University, Taiwan, R.O.C., are also deeply acknowledged.

\section{References}

1. Lu, C. L.; Cui, Z. C.; Wang, Y.; Li, Z.; Guan, C.; Yang, B.; Shen, J. C. J Mater Chem 2003, 13, 2189.

2. Tyan, H. L.; Wei, K. H.; Hsieh, T. H. J. Polym Sci B Pol Phys 2000, 38, 2873.

3. Lee, J. Y.; Lee, H. K. Mater Chem Phys 2004, 85, 410.

4. Dean, K. M.; Bateman, S. A.; Simons, R. Polymer 2007, 48, 2231.

5. Xidas, P. I.; Triantafyllidis, K. S. Eur Polym J 2010, 46, 404.

6. Huang, H. C.; Hsieh, T. E. J Appl Polym Sci 2010, 117, 1252.

7. Jacob, A.; Kurian, P.; Santhosh, A. J Appl Polym Sci 2008, 108, 2623. 
8. Usuki, A.; Kawasumi, M.; Kojima, Y.; Okada, A.; Kurauchi, T.; Kamigaito, O. J Mater Res 1993, 8, 1174.

9. Wang, Y. Y.; Hsieh, T. E. Chem Mater 2005, 17, 3331.

10. Zhu, J.; Morgan, A. B.; Lamelas, F. J.; Wilkie, C. A. Chem Mater 2001, 13, 3774.

11. Wang, W. S.; Chen, H. S.; Wu, Y. W.; Tsai, T. Y.; Chen-Yang, Y. W. Polymer 2008, 49, 4826.

12. Chen, G. X.; Choi, J. B.; Yoon, J. S. Macromol Rapid Commun 2005, 26, 183.

13. de Paiva, L. B.; Morales, A. R.; Díaz, F. R. V. Appl Clay Sci $2008,42,8$.

14. Usuki, A.; Kojima, Y.; Kawasumi, M.; Okada, A.; Fukushima, Y.; Kurauchi, T.; Kamigaito, O. J Mater Res 1993, 8, 1179.

15. Yano, K.; Usuki, A.; Okada, A.; Kurauchi, T.; Kamigaito, O. J Polym Sci A Polym Chem 1993, 31, 2493.

16. Yano, K.; Usuki, A.; Okada, A. J Polym Sci A Polym Chem 1993, 35, 2289.

17. Gilman, J. W.; Jackson, C. L.; Morgan, A. B.; Harris, R.; Manias, E.; Giannelis, E. P.; Wuthenow, M.; Hilton, D.; Phillips, S. H. Chem Mater 2000, 12, 1866.

18. Chen, T. K.; Tien, Y. I.; Wei, K. H. Polymer 2000, 41, 1345.

19. Ceccia, S.; Turcato, E.A.; Maffettone, P. L.; Bongiovanni, R. Prog Org Coat 2008, 63, 110.
20. Benfarhi, S.; Decker, C.; Keller, L.; Zahouily, K. Eur Polym J 2004, 40, 493.

21. Decker, C.; Keller, L.; Zahouily, K.; Benfarhi, S. Polymer 2005, $46,6640$.

22. Uhl, F. M.; Webster, D. C.; Davuluri, S. P.; Wong, S. C. Eur Polym J 2006, 42, 2596.

23. Wang, Y. Y.; Hsieh, T. E. J Mater Sci 2007, 42, 4451.

24. Decker, C.; Viet, T. N. T.; Decker, D.; Weber-Koehl, E. Polymer 2001, 42, 5531.

25. Lee, S. N.; Hwang, S. W.; Chen, C. H. Jpn J Appl Phys 2007, 46, 7432.

26. Chang, K. C.; Chen, S. T.; Lin, H. F.; Lin, C. Y.; Huang, H. H.; Yeh, J. M.; Yu, Y. H. Eur Polym J 2008, 44, 13.

27. Lakshmi, A. S.; Narmadha, B.; Redd, B. S. R. Polym Degrad Stabil 2008, 93, 201.

28. Isci, S.; Uenlue, C. H.; Atici, O. J Appl Polym Sci 2009, 113, 367.

29. Moraes, R. P.; Valera, T. S.; Pereira, A. M. C.; Demarquette, N. R.; Santos, A. M. J Appl Polym Sci 2011, 119, 3658.

30. Choi, Y. Y.; Shin, S. Y. A.; Soares, J. B. P. Macromol Chem Phys 2010, 211, 1026.

31. Kao, K. F.; Lee, C. M.; Chen, M. J.; Tsai, M. J.; Chin, T. S. Adv Mater 2009, 21, 1695.

32. Bharadwaj, R. K. Macromolecules 2001, 34, 9189.

33. Fornes, T. D.; Paul, D. R. Polymer 2003, 44, 4993.

34. Choudalakis, G.; Gotsis, A. D. Eur Polym J 2009, 45, 967. 